\title{
Lyapunov inequalities of left focal $q$-difference boundary value problems and applications
}

\author{
Lulu Zhang ${ }^{1}$, Yige Zhao' and Shurong Sun ${ }^{1 *}$
}

"Correspondence:

sshrong@163.com

${ }^{1}$ School of Mathematical Sciences,

University of Jinan, Jinan, P.R. China

\begin{abstract}
In this paper, we establish some Lyapunov-type inequalities for a class of linear and nonlinear fractional q-difference boundary value problems under Cauchy boundary conditions. As applications, we use the inequality to obtain an interval, where Mittag-Leffler function has no real zeros. In addition, we also derive nonexistence results for fractional q-difference boundary value problem.
\end{abstract}

MSC: 26D15; 65L10; 39A13; 26A33; 33E12

Keywords: Lyapunov inequality; Boundary value problem; Fractional q-difference equations; q-Mittag-Leffler function

\section{Introduction}

The famous Lyapunov inequality [1] states that if a nontrivial solution to the boundary value problem

$$
\begin{aligned}
& u^{\prime \prime}(t)+\lambda(t) u(t)=0, \quad a<t<b, \\
& u(a)=u(b)=0
\end{aligned}
$$

exists, where $\lambda:[a, b] \rightarrow \mathbb{R}$ is a continuous function, then

$$
\int_{a}^{b}|\lambda(s)| d s>\frac{4}{b-a} .
$$

The Lyapunov inequality has many practical applications in differential and difference equations, for example: oscillation theory, disconjugacy, eigenvalue problems, etc.; see [2$12]$ and references therein.

The study of Lyapunov inequalities originated from differential equations, and the research of Lyapunov inequality by constructing fractional differential operators has recently begun. The first work in this direction is due to Ferreira.

\section{Springer}


In 2013, Ferreira [13] considered the following Lyapunov-type inequality for the Riemann-Liouville fractional boundary value problem:

$$
\begin{aligned}
& \left({ }_{a} D^{\alpha} u\right)(t)+q(t) u(t)=0, \quad a<t<b, 1<\alpha \leq 2, \\
& u(a)=u(b)=0,
\end{aligned}
$$

and derived the corresponding Lyapunov-type inequality

$$
\int_{a}^{b}|q(s)| d s>\Gamma(\alpha)\left(\frac{4}{b-a}\right)^{\alpha-1} .
$$

In 2014, Ferreira [14] derived the corresponding Lyapunov-type inequality for a differential equation that depends on the Caputo fractional derivative, i.e., for the boundary value problem

$$
\begin{aligned}
& { }_{a}^{c} D^{\alpha} u(t)+q(t) u(t)=0, \quad a<t<b, 1<\alpha \leq 2, \\
& u(a)=u(b)=0 .
\end{aligned}
$$

If $u$ is a nontrivial continuous solution to the above problem, then

$$
\int_{a}^{b}|q(s)| d s>\frac{\Gamma(\alpha) \alpha^{\alpha}}{[(\alpha-1)(b-a)]^{\alpha-1}} .
$$

There are many articles about the boundary value problem of fractional $q$-difference equation as follows.

In 2011, Ferreira [15] studied the existence of positive solutions to the nonlinear $q$ difference boundary value problem

$$
\begin{array}{ll}
D_{q}^{\alpha} u(t)+f(u(t))=0, & 0<t<1,2<\alpha \leq 3, \\
u(0)=D_{q} u(0)=0, & D_{q} u(1)=\beta \geq 0 .
\end{array}
$$

By using a fixed point theorem in a cone, El-Shahed and Al-Askar [16] were concerned with the existence of positive solutions to the nonlinear $q$-difference equation

$$
\begin{aligned}
& { }_{c} D_{q}^{\alpha} u(t)+a(t) f(u(t))=0, \quad 0<t \leq 1,2<\alpha \leq 3, \\
& u(0)=D_{q}^{2} u(0) \leq 0, \quad a D_{q} u(1)+b D_{q}^{2} u(1)=0,
\end{aligned}
$$

where $a, b \geq 0$ and ${ }_{c} D_{q}^{\alpha}$ is the fractional $q$-derivatives of the Caputo type.

Recently, Liang and Zhang [17] discussed the following nonlinear $q$-fractional threepoint boundary value problem:

$$
\begin{aligned}
& \left(D_{q}^{\alpha} u\right)(t)+f(t, u(t))=0, \quad 0<t<1,2<\alpha \leq 3, \\
& u(0)=\left(D_{q} u\right)(0)=0, \quad D_{q} u(1)=\beta D_{q} u(\eta) .
\end{aligned}
$$

By using a fixed point theorem in partially ordered sets, the authors obtained sufficient conditions for the existence and uniqueness of positive and nondecreasing solutions to the above boundary value problem. 
To the best of our knowledge, there are few papers which consider the Lyapunov-type inequality for a fractional $q$-difference boundary value problem.

In 2016, M. Jleli and B. Samet [18] considered the following fractional $q$-difference equation:

$$
\left({ }_{a} D_{q}^{\alpha} u\right)(t)+\varphi(t) u(t)=0, \quad a<t<b, q \in[0,1), 1<\alpha \leq 2,
$$

subject to Dirichlet-type boundary conditions

$$
u(a)=u(b)=0 .
$$

If $u$ is a nontrivial continuous solution to the fractional $q$-difference boundary value problem, then

$$
\int_{a}^{b}(s-a)^{\alpha-1}(b-(q s+(1-q) a))_{a}^{(\alpha-1)}|\varphi(s)|_{a} d_{q} s \geq \Gamma_{q}(\alpha)(b-a)^{\alpha-1},
$$

where ${ }_{a} D^{\alpha}$ denotes the fractional $q$-derivative of Riemann-Liouville type and $\varphi:[a, b] \rightarrow$ $\mathbb{R}$ is a continuous function.

In 2018, K. Ma and S. Sun [19] considered the nonlinear fractional $q$-difference equations with Dirichlet-type boundary conditions

$$
\begin{aligned}
& D_{q}^{\alpha} u(t)+h(t) f(u(t))=0, \quad 0<t<1, \\
& u(0)=u(1)=0 .
\end{aligned}
$$

If the fractional boundary value problem has a nontrivial solution $u$, then

$$
\int_{0}^{1}|h(t)|[q t(1-q t)]^{(\alpha-1)} d_{q} t \geq \frac{\Gamma_{q}(\alpha) \eta}{f(\eta)}
$$

where $\eta=\max _{0 \leq t \leq 1} u(t)$.

In this work, we consider the following boundary value problem with Cauchy-type boundary condition:

$$
\begin{aligned}
& \left(D_{q}^{\alpha} u\right)(t)+\lambda(t) u(t)=0, \quad a<t<b, 0 \leq q<1,2<\alpha \leq 3, \\
& \left(D_{q}^{\alpha} u\right)(t)+\lambda(t) f(u(t))=0, \quad a<t<b, 0 \leq q<1,2<\alpha \leq 3,
\end{aligned}
$$

subject to boundary conditions

$$
D_{q} u(a)=0, \quad u(b)=0, \quad u(a)=0,
$$

where ${ }_{a} D_{q}^{\alpha}$ denotes the fractional $q$-derivative of Riemann-Liouville type of order $\alpha$, and $\lambda:[a, b] \rightarrow \mathbb{R}$ is a continuous function.

A Cauchy-type boundary condition specifies both the function value and normal derivative on the boundary of the domain. This corresponds to imposing both Dirichlet and Neumann boundary conditions. In addition, this boundary condition is simple and common, therefore, in this paper, we make full use of the boundary condition. 
The innovation of this paper is to consider the Lyapunov-type inequality of linear and nonlinear fractional $q$-difference equations under Cauchy boundary conditions, to investigate the existence interval of $q$-Mittag-Leffler function with no real zeros, and to take advantage of Laplace transform, which most papers rarely consider.

The article is arranged as follows. In Sect. 2, we list some basic definitions about Riemann-Liouville fractional calculus, together with some basic properties and lemmas to prove our main results. In Sect. 3, we get some main results and several corollaries. In Sect. 4, we use the inequality to obtain an interval, where Mittag-Leffler function has no real zeros. In addition, we consider Lyapunov-type inequalities of nonlinear fractional $q$-difference equations.

\section{Basic definitions and preliminaries}

In this section, we list some useful definitions and preliminaries, which are helpful for the proof of the main results. These materials can be found in the recent literature, see [20,21].

For $q \in(0,1)$, we define

$$
[a]_{q}=\frac{1-q^{a}}{1-q}, \quad a \in \mathbb{R} .
$$

The $q$-analogue of the power function $(a-b)^{k}$ with $k \in \mathbb{N}_{0}:=\{0,1,2, \ldots\}$ is

$$
(a-b)^{0}=1, \quad(a-b)^{(k)}=\prod_{i=0}^{k-1}\left(a-b q^{i}\right), \quad k \in \mathbb{N}, a, b \in \mathbb{R} .
$$

More generally, if $\gamma \in \mathbb{R}$, then

$$
(a-b)^{(\gamma)}=a^{\gamma} \prod_{i=0}^{\infty} \frac{a-b q^{i}}{a-b q^{i+r}}, \quad a \neq 0 .
$$

Note if $b=0$, then $a^{(\gamma)}=a^{\gamma}$. We also use the notation $0^{(\gamma)}=0$ for $\gamma \geq 0$. Here we point that the following equality holds:

$$
(a-b)^{(\gamma)}=\left(a-b q^{\gamma-1}\right)(a-b)^{(\gamma-1)} .
$$

Definition 2.1 ([20]) Let $\alpha \geq 0$ and $f$ be a real function defined on a certain interval $[a, b]$. The Riemann-Liouville fractional $q$-integral of order $\alpha$ is defined by

$$
\begin{aligned}
& \left(I_{q}^{0} f\right)(t)=f(t), \\
& \left(I_{q}^{\alpha} f\right)(t)=\frac{1}{\Gamma_{q}(\alpha)} \int_{0}^{t}(t-q s)^{(\alpha-1)} f(s) d_{q} s, \quad \alpha>0, t \in[a, b] .
\end{aligned}
$$

Definition 2.2 ([20]) The fractional $q$-derivative of order $\alpha>0$ of a continuous and differentiable function $f$ is given by

$$
\begin{aligned}
& \left(D_{q}^{0} f\right)(t)=f(t), \quad t \in[a, b], \\
& \left(D_{q}^{\alpha} f\right)(t)=\left(D_{q}^{l} I_{q}^{l-\alpha} f\right)(t), \quad \alpha>0, t \in[a, b],
\end{aligned}
$$

where $l$ is the smallest integer greater than or equal to $\alpha$. 
Lemma 2.1 ([21]) Let $\alpha, \beta \geq 0$ and $f:[a, b] \rightarrow \mathbb{R}$ be a continuous function defined on $[0,1]$, and assume its derivative exists. Then, the following formulas hold:

$$
\begin{aligned}
& D_{q}^{\alpha}\left(I_{q}^{\alpha} f\right)(t)=f(t), \\
& I_{q}^{\alpha} I_{q}^{\beta} f(t)=I_{q}^{\alpha+\beta} f(t) .
\end{aligned}
$$

Lemma 2.2 ([21]) Let $f:[a, b] \rightarrow \mathbb{R}$ be differentiable, $p$ be a positive integer and $\alpha>p-1$.

Then

$$
I_{q}^{\alpha} D_{q}^{p} f(t)=D_{q}^{p} I_{q}^{\alpha} f(t)-\sum_{k=0}^{p-1} \frac{(t-a)^{(\alpha-p+k)}}{\Gamma_{q}(\alpha+k-p+1)} D_{q}^{k} f(a), \quad t \in[a, b] .
$$

Lemma 2.3 ([21]) The first order q-Leibniz rule for the functions $f$ and $g$ is given by

$$
D_{q}(f g)(t)=f(t)\left(D_{q} g\right)(t)+g(q t)\left(D_{q} f\right)(t) .
$$

\section{Lyapunov-type inequalities of linear fractional q-difference equation}

At first, we need an integral representation of the solution to problem (1.1) and (1.3).

Lemma 3.1 Function $u \in[a, b]$ is a solution to (1.1) and (1.3) if and only if $u$ is a solution to the integral equation

$$
u(t)=\int_{a}^{b} G(t, q s) \lambda(s) u(s) d_{q} s, \quad t \in[a, b]
$$

in which $G$, the Green function linking (1.1) and (1.3), is given by

$$
G(t, q s)=\frac{1}{\Gamma_{q}(\alpha)} \begin{cases}(b-q s)^{(\alpha-1)}\left(\frac{t-a}{b-a}\right)^{(\alpha-1)}-(t-q s)^{(\alpha-1)}, & a \leq q s \leq t \leq b, \\ (b-q s)^{(\alpha-1)}\left(\frac{t-a}{b-a}\right)^{(\alpha-1)}, & a \leq t \leq q s \leq b .\end{cases}
$$

Proof By making use of Lemmas 2.1 and 2.2 with $p=3$, from (1.1), we can see that

$$
\left({ }_{a} D_{q}^{\alpha} u\right)(t)=-\lambda(t) u(t)
$$

so

$$
\left(I_{q}^{\alpha} D_{q}^{3} I_{q}^{3-\alpha} u\right)(t)=-I_{q}^{\alpha} \lambda(t) u(t)
$$

i.e.,

$$
\left(D_{q}^{3} I_{q}^{\alpha} I_{q}^{3-\alpha} u\right)(t)=-I_{q}^{\alpha} \lambda(t) u(t)
$$

We can get a general solution to (1.1) as

$$
\begin{aligned}
u(t)= & c_{1}(t-a)^{(\alpha-1)}+c_{2}(t-a)^{(\alpha-2)}+c_{3}(t-a)^{(\alpha-3)} \\
& -\frac{1}{\Gamma_{q}(\alpha)} \int_{a}^{t}(t-q s)^{(\alpha-1)} \lambda(s) u(s) d_{q} s
\end{aligned}
$$


in which $c_{1}, c_{2}, c_{3}$ are real constants. Due to the boundary value conditions $u(a)=0$, we know that $c_{3}=0$. Taking the $q$-derivative of $u(t)$, we get

$$
\begin{aligned}
D_{q} u(t)= & {[\alpha-1]_{q} c_{1}(t-a)^{(\alpha-2)}+[\alpha-2]_{q} c_{2}(t-a)^{(\alpha-3)} } \\
& -\frac{1}{\Gamma_{q}(\alpha)} \int_{a}^{t}[\alpha-1]_{q}(t-q s)^{(\alpha-2)} \lambda(s) u(s) d_{q} s .
\end{aligned}
$$

By using the boundary condition $D_{q} u(a)=0$, we get

$$
c_{2}=0 .
$$

Then the boundary condition given by (1.2) yields

$$
c_{1}(b-a)^{(\alpha-1)}-\frac{1}{\Gamma_{q}(\alpha)} \int_{a}^{b}(b-q s)^{(\alpha-1)} \lambda(s) u(s) d_{q} s=0,
$$

so

$$
c_{1}=\frac{\int_{a}^{b}(b-q s)^{(\alpha-1)} \lambda(s) u(s) d_{q} s}{\Gamma_{q}(\alpha)(b-a)^{(\alpha-1)}} .
$$

Therefore

$$
\begin{aligned}
u(t)= & \frac{\int_{a}^{b}(b-q s)^{(\alpha-1)} \lambda(s) u(s) d_{q} s}{\Gamma_{q}(\alpha)(b-a)^{(\alpha-1)}}(t-a)^{(\alpha-1)}-\frac{1}{\Gamma_{q}(\alpha)} \int_{a}^{t}(t-q s)^{(\alpha-1)} \lambda(s) u(s) d_{q} s \\
= & \frac{1}{\Gamma_{q}(\alpha)} \int_{a}^{t}\left[(b-q s)^{(\alpha-1)}\left(\frac{t-a}{b-a}\right)^{(\alpha-1)}-(t-q s)^{(\alpha-1)}\right] \lambda(s) u(s) d_{q} s \\
& +\frac{1}{\Gamma_{q}(\alpha)} \int_{t}^{b}\left[(b-q s)^{(\alpha-1)}\left(\frac{t-a}{b-a}\right)^{(\alpha-1)}\right] \lambda(s) u(s) d_{q} s \\
= & \int_{a}^{b} G(t, q s) \lambda(s) u(s) d_{q} s .
\end{aligned}
$$

The sufficiency is obvious, so we obtain the desired result.

The properties of Green function play an important role in this paper.

Lemma 3.2 Green function $G$ defined above has the following properties:

(1) $G(t, q s) \geq 0, a \leq t, q s \leq b$;

(2) $\max _{t \in[a, b]} G(t, q s)=G(q s, q s), q s \in[a, b]$;

(3) $G(q s, q s)$ has a unique maximum given by

$$
\max _{q s \in[a, b]} G(q s, q s)=G\left(\frac{a+b}{2}, \frac{a+b}{2}\right)=\frac{1}{\Gamma_{q}(\alpha)}\left(\frac{b-a}{4}\right)^{(\alpha-1)} .
$$


Proof (1) Let

$$
\begin{aligned}
& g_{1}(t, q s)=(b-q s)^{(\alpha-1)}\left(\frac{t-a}{b-a}\right)^{(\alpha-1)}-(t-q s)^{(\alpha-1)}, \quad a \leq q s \leq t \leq b, \\
& g_{2}(t, q s)=(b-q s)^{(\alpha-1)}\left(\frac{t-a}{b-a}\right)^{(\alpha-1)}, \quad a \leq t \leq q s \leq b .
\end{aligned}
$$

When $a \leq t \leq q s \leq b$, it is clear that $g_{2}(t, q s)>0$.

When $a \leq q s \leq t \leq b$,

$$
\begin{aligned}
g_{1}(t, q s) & =(b-q s)^{(\alpha-1)}\left(\frac{t-a}{b-a}\right)^{(\alpha-1)}-(t-q s)^{(\alpha-1)} \\
& =\left(\frac{t-a}{b-a}\right)^{(\alpha-1)}(b-q s)^{(\alpha-1)}-\left(\frac{t-a}{b-a}\right)^{(\alpha-1)}\left(b-\left(a+\frac{(q s-a)(b-a)}{t-a}\right)\right)^{(\alpha-1)} .
\end{aligned}
$$

Observe now that

$$
a+\frac{(q s-a)(b-a)}{t-a} \geq q s
$$

if and only if

$$
(q s-a)(b-a) \geq(q s-a)(t-a), \quad a \leq q s \leq t \leq b,
$$

so $g_{1}(t, q s) \geq 0$. Therefore, $G(t, q s) \geq 0$ for all $a \leq t, q s \leq b$.

(2) From (1), we obtain $\max _{t \in[a, b]} G(t, q s)=G(q s, q s)$, qs $\in[a, b]$.

(3) Now we obtain the maximum of $G(q s, q s)$.

According to Lemma 2.3, we get

$$
\begin{aligned}
D_{q} G(q s, q s) & =D_{q}\left[\left(\frac{q s-a}{b-a}\right)^{(\alpha-1)}(b-q s)^{(\alpha-1)}\right] \\
& =[\alpha-1]_{q} \frac{(q s-a)^{(\alpha-2)}}{(b-a)^{(\alpha-1)}}(b-q s)^{(\alpha-2)}(-2 q s+b+a),
\end{aligned}
$$

which implies that $D_{q} G(q s, q s)=0$ only at $q s=\frac{b+a}{2}$ and $D_{q} G(q s, q s)>0$ for $q s<\frac{b+a}{2}$ and $D_{q} G(q s, q s)<0$ for $q s>\frac{b+a}{2}$.

From what has been discussed above, the maximum of $G(t, q s)$ is $G\left(\frac{a+b}{2}, \frac{a+b}{2}\right)$, which results in $\frac{1}{\Gamma_{q}(\alpha)}\left(\frac{b-a}{4}\right)^{(\alpha-1)}$. This completes the proof.

Theorem 3.1 If a nontrivial continuous solution to the fractional q-difference boundary value problem

$$
\begin{aligned}
& \left(D_{q}^{\alpha} u\right)(t)+\lambda(t) u(t)=0, \quad a<t<b, q \in[0,1), 2<\alpha \leq 3, \\
& D_{q} u(a)=0, \quad u(a)=0, \quad u(b)=0
\end{aligned}
$$

exists, where $\lambda:[a, b] \rightarrow \mathbb{R}$ is a continuous function, then

$$
\int_{a}^{b}|\lambda(s)|(b-q s)^{(\alpha-1)}(q s-a)^{(\alpha-1)} d_{q} s \geq \Gamma_{q}(\alpha)(b-a)^{(\alpha-1)} .
$$


Proof Let $u$ be a nontrivial continuous solution to the fractional $q$-difference boundary value problem (3.1). Let

$$
\|u\|=\max _{a \leq t \leq b}|u| .
$$

By Lemma 3.1, for all $a \leq t \leq b$, we have

$$
\begin{aligned}
|u(t)| & \leq \int_{a}^{b} G(q s, q s)|\lambda(s)||u(s)| d_{q} s \\
& \leq\|u\| \int_{a}^{b} G(q s, q s)|\lambda(s)| d_{q} s
\end{aligned}
$$

if and only if

$$
1 \leq \int_{a}^{b} G(q s, q s)|\lambda(s)| d_{q} s
$$

Hence,

$$
\int_{a}^{b}|\lambda(s)|(b-q s)^{(\alpha-1)}(q s-a)^{(\alpha-1)} d_{q} s \geq \Gamma_{q}(\alpha)(b-a)^{(\alpha-1)},
$$

which completes the proof.

Corollary 3.1 If a nontrivial continuous solution to the Riemann-Liouville fractional boundary value problem

$$
\begin{aligned}
& \left(D^{\alpha} u\right)(t)+\lambda(t) u(t)=0, \quad a<t<b, 2<\alpha \leq 3, \\
& D u(a)=0, \quad u(a)=0, \quad u(b)=0
\end{aligned}
$$

exists, where $\lambda:[a, b] \rightarrow \mathbb{R}$ is a continuous function and ${ }_{a} D^{\alpha}$ denotes the RiemannLiouville fractional derivative of order $\alpha$, then

$$
\int_{a}^{b}|\lambda(s)|(b-s)^{\alpha-1}(s-a)^{\alpha-1} d s \geq \Gamma(\alpha)(b-a)^{\alpha-1} .
$$

Proof It follows from Theorem 3.1 by letting $q \rightarrow 1^{-}$.

Corollary 3.2 If there exists a nontrivial continuous solution of the fractional q-difference boundary value problem

$$
\begin{aligned}
& D_{q}^{\alpha} u(t)+\lambda(t) u(t)=0, \quad a<t<b, q \in[0,1), 2<\alpha \leq 3, \\
& D_{q} u(a)=0, \quad u(a)=0, \quad u(b)=0,
\end{aligned}
$$

where $\lambda:[a, b] \rightarrow \mathbb{R}$ is a continuous function, then

$$
\int_{a}^{b}|\lambda(s)| d_{q} s \geq \Gamma_{q}(\alpha)\left(\frac{4}{b-a}\right)^{(\alpha-1)}
$$


Proof Let $u$ be a nontrivial continuous solution to the fractional boundary value problem (3.1). Let

$$
\|u\|=\max _{a \leq t \leq b}|u(t)| .
$$

By Lemma 3.1, for all $a \leq t \leq b$, we have

$$
u(t)=\int_{a}^{b} G(t, q s) \lambda(s) u(s) d_{q} s, \quad t \in[a, b] .
$$

Then

$$
|u(t)| \leq\|u\| \max _{a \leq t \leq b} \int_{a}^{b} G(t, q s)|\lambda(s)| d_{q} s .
$$

Therefore

$$
\begin{aligned}
& |u(t)| \leq\|u\| \frac{1}{\Gamma_{q}(\alpha)}\left(\frac{b-a}{4}\right)^{(\alpha-1)} \int_{a}^{b}|\lambda(s)| d_{q} s, \\
& 1 \leq \frac{1}{\Gamma_{q}(\alpha)}\left(\frac{b-a}{4}\right)^{(\alpha-1)} \int_{a}^{b}|\lambda(s)| d_{q} s,
\end{aligned}
$$

and as a consequence,

$$
\int_{a}^{b}|\lambda(s)| d_{q} s \geq \Gamma_{q}(\alpha)\left(\frac{4}{b-a}\right)^{(\alpha-1)},
$$

which yields the desired inequality. In fact, this conclusion is a generalization of Theorem 3.1.

Corollary 3.3 If a nontrivial continuous solution to the Riemann-Liouville fractional boundary value problem

$$
\begin{aligned}
& \left(D^{\alpha} u\right)(t)+\lambda(t) u(t)=0, \quad a<t<b, 2<\alpha \leq 3, \\
& D_{q} u(a)=0, \quad u(a)=0, \quad u(b)=0
\end{aligned}
$$

exists, where $\lambda:[a, b] \rightarrow \mathbb{R}$ is a continuous function, then

$$
\int_{a}^{b}|\lambda(s)| d s \geq \Gamma(\alpha)\left(\frac{4}{b-a}\right)^{\alpha-1} .
$$

Proof Let $q \rightarrow 1^{-}$in Corollary 3.2.

Corollary 3.4 If a nontrivial continuous solution to the boundary value problem

$$
\begin{aligned}
& u^{\prime \prime}(t)+\lambda(t) u(t)=0, \quad a<t<b, \\
& u^{\prime}(a)=0, \quad u(a)=0, \quad u(b)=0
\end{aligned}
$$


exists, where $\lambda:[a, b] \rightarrow \mathbb{R}$ is a continuous function, then

$$
\int_{a}^{b}|\lambda(s)| d s \geq \frac{4}{b-a}
$$

Proof We need take $\alpha=2$ in the Corollary 3.3, which immediately gives us the proof.

Consider the following problem:

$$
D_{q}^{\alpha} u(t)+\lambda(t) u(t)=0, \quad 0<t<1
$$

subject to the boundary value problem

$$
D_{q} u(0)=0, \quad u(0)=0, \quad u(1)=0,
$$

where $\lambda:[0,1] \rightarrow \mathbb{R}$ is a continuous function. We have the following result:

Corollary 3.5 Assume that

$$
\int_{0}^{1}|\lambda(s)| d_{q} s<4^{(\alpha-1)} \Gamma_{q}(\alpha)
$$

Then (3.8)-(3.9) has no nontrivial solution.

Proof Assume on the contrary that (3.8)-(3.9) has a nontrivial solution. By Corollary 3.2, we get $\int_{0}^{1}|\lambda(s)| d_{q} s \geq 4^{(\alpha-1)} \Gamma_{q}(\alpha)$, which contradicts assumption (3.10).

\section{On real zeros of the $q$-Mittag-Leffler function}

In this section, we use the Lyapunov inequality in Theorem 3.1 to obtain an interval, where $q$-Mittag-Leffler function has no real zeros.

Definition 4.1 ([22]) Let $\alpha>0, \beta \in \mathbb{C}$, then the function

$$
e_{\alpha, \beta}(z ; q):=\sum_{n=0}^{\infty} \frac{z^{n}}{\Gamma_{q}(n \alpha+\beta)}, \quad\left(\left|z(1-q)^{\alpha}\right|<1\right)
$$

is called the $q$-Mittag-Leffler function.

Lemma 4.1 ([22]) Let $\alpha, \beta, a \in \mathbb{R}^{+}$and $k \in \mathbb{N}$. Then the identity

$$
{ }_{q} \mathfrak{L}\left(t^{k \alpha+\beta-1} e_{\alpha, \beta}^{(k)}\left( \pm a t^{\alpha} ; q\right)\right)=\frac{p^{\alpha-\beta}}{1-q} \frac{k !}{\left(p^{\alpha} \mp a\right)^{k+1}}, \quad|p|^{\alpha}>a
$$

is valid in the disk $\left\{t \in \mathcal{C}: a|t(1-q)|^{\alpha}<1\right\}$.

Theorem 4.1 Let $2<\alpha \leq 3$. Then the q-Mittag-Leffler function $e_{\alpha, \alpha}(z)$ has no real zeros for

$$
z \in\left(-\infty, \frac{\Gamma_{q}(\alpha)}{\int_{0}^{1}(1-q s)^{(\alpha-1)}(q s)^{(\alpha-1)} d_{q} s}\right) .
$$


Proof Let $a=0, b=1$ and consider the following fractional $q$-difference equations:

$$
\begin{aligned}
& D_{q}^{\alpha} u(t)+\lambda u(t)=0, \quad 0<t<1, q \in[0,1), 2<\alpha \leq 3, \\
& u(1)=0, \quad D_{q} u(0)=0, \quad u(0)=0 .
\end{aligned}
$$

We can use Laplace transform for (4.2) to obtain

$$
\begin{aligned}
{ }_{q} \mathfrak{L} D_{q}^{\alpha} u(t)= & \frac{s^{\alpha}}{(1-q)^{\alpha}} q \mathfrak{L} u(t)-D_{q}^{\alpha-1} u(0) \frac{1}{1-q} \\
& -D_{q}^{\alpha-2} u(0) \frac{s}{(1-q)^{2}}-D_{q}^{\alpha-3} u(0) \frac{s^{2}}{(1-q)^{3}} \\
= & -{ }_{q} \mathfrak{L} \lambda u(t) .
\end{aligned}
$$

Therefore, we get

$$
\left\{\frac{s^{\alpha}}{(1-q)^{\alpha}}+\lambda\right\}_{q} \mathfrak{L} u(t)=\frac{D_{q}^{\alpha-1} u(0)}{1-q}+\frac{D_{q}^{\alpha-2} u(0) s}{(1-q)^{2}}+\frac{D_{q}^{\alpha-3} u(0) s^{2}}{(1-q)^{3}} .
$$

Thus

$$
{ }_{q} \mathfrak{L} u(t)=\frac{\frac{D_{q}^{\alpha-1} u(0)}{1-q}+\frac{D_{q}^{\alpha-2} u(0) s}{(1-q)^{2}}+\frac{D_{q}^{\alpha-3} u(0) s^{2}}{(1-q)^{3}}}{\frac{s^{\alpha}}{(1-q)^{\alpha}}+\lambda} .
$$

According to Lemma 4.1, we suppose $k=0, a=\lambda, p=3$ and $\beta=\alpha, \alpha-1, \alpha-2$, respectively. We can conclude that

$$
u(t)=C_{1} t^{\alpha-1} e_{\alpha, \alpha}^{(0)}\left(-\lambda t^{\alpha} ; q\right)+C_{2} t^{\alpha-2} e_{\alpha, \alpha-1}^{(0)}\left(-\lambda t^{\alpha} ; q\right)+C_{3} t^{\alpha-3} e_{\alpha, \alpha-2}^{(0)}\left(-\lambda t^{\alpha} ; q\right) .
$$

In the expression of $u(t)$, we take $C_{1}=1, C_{2}=C_{3}=0$, thus getting a solution to this equation:

$$
u(t)=t^{\alpha-1} e_{\alpha, \alpha}^{(0)}\left(-\lambda t^{\alpha} ; q\right)
$$

If $\lambda \in \mathbb{R}$ is an eigenvalue of the boundary value problem of (4.2), then

$$
e_{\alpha, \alpha}^{(0)}(-\lambda)=0,
$$

and the corresponding eigenfunction is given by

$$
u(t)=t^{\alpha-1} e_{\alpha, \alpha}^{(0)}\left(-\lambda t^{\alpha}\right)
$$

According to Theorem 3.1, if $\lambda \in \mathbb{R}$ is an eigenvalue of (4.2), i.e., $-\lambda$ is a zero of equation (4.8), then

$$
\lambda \geq \frac{\Gamma_{q}(\alpha)}{\int_{0}^{1}(1-q s)^{(\alpha-1)}(q s)^{(\alpha-1)} d_{q} s},
$$

which concludes the proof. 


\section{Lyapunov-type inequalities of nonlinear fractional $q$-difference equations}

In this section, we consider a nonlinear fractional $q$-difference equation.

Consider the boundary value problem

$$
\left(D_{q}^{\alpha} u\right)(t)+\lambda(t) f(u(t))=0, \quad a<t<b, q \in[0,1), 2<\alpha \leq 3
$$

subject to the boundary conditions

$$
D_{q} u(a)=0, \quad u(a)=0, \quad u(b)=0,
$$

where ${ }_{a} D_{q}^{\alpha}$ denotes the fractional $q$-derivative of Riemann-Liouville type of order $\alpha, 2<$ $\alpha \leq 3$ and $f:[a, b] \times \mathbb{R} \rightarrow \mathbb{R}$.

By $\mathbf{B}=C([a, b], \mathbb{R})$ we denote the Banach space of all continuous functions from $[a, b]$ into $\mathbb{R}$ with the norm $\|u\|=\max _{a \leq t \leq b}|u(t)|$. And by $\mathbf{L}[a, b]$ we denote the space of all real functions defined on $[a, b]$, which are Lebesgue integrable with the norm $\|u\|_{L}=$ $\int_{a}^{b}|u(s)| d_{q} s$.

Lemma 5.1 ([19], Jensen's inequality) Let $u \in C([a, b],(c, d))$ and $\lambda:[a, b] \rightarrow \mathbb{R}$ be a real Lebesgue integrable function with $\int_{a}^{b}|\lambda(s)| d_{q} s>0$, where $a, b, c, d \in \mathbb{R}$. If $f \in((c, d), \mathbb{R})$ is concave, then

$$
f\left(\frac{\int_{a}^{b}|\lambda(s)| u(s) d_{q} s}{\int_{a}^{b}|\lambda(s)| d_{q} s}\right) \geq \frac{\int_{a}^{b}|\lambda(s)| f(u(s)) d_{q} s}{\int_{a}^{b}|\lambda(s)| d_{q} s} .
$$

Remark 5.1 If $f$ is a convex, then the above inequality holds with " $\geq$ " substituted by " $\leq$ ".

Theorem 5.1 Let $\lambda:[a, b] \rightarrow \mathbb{R}$ be a real nontrivial Lebesgue integrable function. Assume that $f \in C\left(\mathbb{R}_{+}, \mathbb{R}_{+}\right)$is a concave and nondecreasing function. If the fractional boundary value problem

$$
\begin{aligned}
& \left(D_{q}^{\alpha} u\right)(t)+\lambda(t) f(u(t))=0, \quad a<t<b, \\
& D_{q} u(a)=0, \quad u(a)=0, \quad u(b)=0
\end{aligned}
$$

has a nontrivial solution $u$, then

$$
\int_{a}^{b}|\lambda(s)| d_{q} s \geq \frac{4^{(\alpha-1)} \Gamma_{q}(\alpha) \xi}{(b-a)^{(\alpha-1)} f(\xi)}
$$

where $\xi=\max _{a \leq t \leq b} u(t)$.

Proof According to Lemma 3.1, we know that

$$
|u(t)| \leq \int_{a}^{b} G(t, q s)|\lambda(s)| f(u(s)) d_{q} s
$$


and, by using Jensen's inequality [19] and since $f$ is concave and nondecreasing, we get that

$$
\begin{aligned}
\|u\| & \leq \int_{a}^{b} G(q s, q s)|\lambda(s)| f(u(s)) d_{q} s \\
& \leq \frac{\|\lambda\|_{L}(b-a)^{(\alpha-1)}}{\Gamma_{q}(\alpha) 4^{(\alpha-1)}} \int_{a}^{b} \frac{|\lambda(s)| f(u(s))}{\|\lambda\|_{L}} d_{q} s \\
& \leq \frac{\|\lambda\|_{L}(b-a)^{(\alpha-1)}}{\Gamma_{q}(\alpha) 4^{(\alpha-1)}} f(\xi),
\end{aligned}
$$

where $\xi=\max _{a \leq t \leq b} u(t)$. Therefore,

$$
\int_{a}^{b}|\lambda(s)| d_{q} s \geq \frac{4^{(\alpha-1)} \Gamma_{q}(\alpha) \xi}{(b-a)^{(\alpha-1)} f(\xi)}
$$

This completes the proof.

Corollary 5.1 If the Riemann-Liouville fractional boundary value problem

$$
\begin{array}{ll}
\left(D^{\alpha} u\right)(t)+\lambda(t) f(u(t))=0, & a<t<b, 2<\alpha \leq 3, \\
D u(a)=0, \quad u(a)=0, & u(b)=0
\end{array}
$$

has a nontrivial solution $u$, where $\lambda:[a, b] \rightarrow \mathbb{R}$ is a continuous function, then

$$
\int_{a}^{b}|\lambda(s)| d s \geq \frac{4^{\alpha-1} \Gamma(\alpha) \xi}{(b-a)^{\alpha-1} f(\xi)}
$$

where $\xi=\max _{a \leq t \leq b} u(t)$.

Proof It follows from Theorem 5.1 by letting $q \rightarrow 1^{-}$.

Corollary 5.2 If a nontrivial continuous solution to the Riemann-Liouville fractional boundary value problem

$$
\begin{aligned}
& \left(D_{q}^{3} u\right)(t)+\lambda(t) f(u(t))=0, \quad a<t<b, 2<\alpha \leq 3, \\
& D_{q} u(a)=0, \quad u(a)=0, \quad u(b)=0
\end{aligned}
$$

exists, where $\lambda:[a, b] \rightarrow \mathbb{R}$ is a real nontrivial Lebesgue integrable function and $f \in C(\mathbb{R}, \mathbb{R})$ is a concave and nondecreasing function, then

$$
\int_{a}^{b}|\lambda(s)| d s \geq \frac{32 \xi}{(b-a)^{2} f(\xi)}
$$

where $\xi=\max _{a \leq t \leq b} u(t)$.

Proof From Corollary 5.1 by taking $\alpha=3$, we get

$$
\int_{a}^{b}|\lambda(s)| d s \geq \frac{4^{2} \Gamma(3) \xi}{(b-a)^{2} f(\xi)}=\frac{(16 \times 2) \xi}{(b-a)^{2} f(\xi)}=\frac{32 \xi}{(b-a)^{2} f(\xi)}
$$

which completes the proof. 


\section{Acknowledgements}

The authors sincerely thank the reviewers for their valuable suggestions and useful comments that have led to the present improved version of the original manuscript.

\section{Funding}

This research is supported by the Natural Science Foundation of China (61703180, 61803176), and supported by Shandong Provincial Natural Science Foundation (ZR2016AM17, ZR2017MA043),

\section{Competing interests}

The authors declare that they have no competing interests.

\section{Authors' contributions}

The authors declare that the study was realized in collaboration with the same responsibility. All authors read and approved the final manuscript.

\section{Publisher's Note}

Springer Nature remains neutral with regard to jurisdictional claims in published maps and institutional affiliations.

Received: 7 July 2018 Accepted: 8 February 2019 Published online: 04 April 2019

\section{References}

1. Liapunov, A.M.: Problèm général de la stabilité du mouvement. Ann. Fac. Sci. Toulouse 2, 203-474 (1907)

2. Clark, S., Hinton, D.B.: A Lyapunov inequality for linear Hamiltonian systems. Math. Inequal. Appl. 1, $201-209$ (1998)

3. Cakmak, D.: Lyapunov-type integral inequalities for certain higher order differential equations. Appl. Math. Comput. 216, 368-373 (2010)

4. Zhang, M., Tang, X.H.: Lyapunov inequalities and stability for discrete linear Hamiltonian systems. Appl. Math. Comput. 218, 574-582 (2011)

5. Liu, X., Tang, M.: Lyapunov-type inequality for higher order difference equations. Appl. Math. Comput. 232, 666-669 (2014)

6. Yang, X.: On Lyapunov-type inequality for certain higher-order differential equations. Appl. Math. Comput. 134 307-317 (2003)

7. Yang, X., Lo, K.: Lyapunov-type inequality for a class of even-order differential equations. Appl. Math. Comput. 215 3884-3890 (2010)

8. Bai, Z.B., Zhang, S., Sun, S.J., Yin, C.: Monotone iterative method for a class of fractional differential equations. Electron. J. Differ. Equ. 2016, 6 (2016)

9. Zhang, X., Zhong, Q.: Uniqueness of solution for higher-order fractional differential equations with conjugate type integral conditions. Fract. Calc. Appl. Anal. 20(6), 1471-1484 (2017)

10. Cui, Y.J., Sun, Q., Su, X.W.: Monotone iterative technique for nonlinear boundary value problems of fractional order $p \in(2,3]$. Adv. Differ. Equ. 2017, 248 (2017)

11. Zhang, X., Zhong, Q.: Triple positive solutions for nonlocal fractional differential equations with singularities both on time and space variables. Appl. Math. Lett. 80, 12-19 (2018)

12. Cui, Y., Ma, W.J., Sun, Q., Su, X.W.: New uniqueness results for boundary value problem of fractional differential equation. Nonlinear Anal. 223(1), 31-39 (2018)

13. Ferreira, R.A.C.: A Lyapunov-type inequality for a fractional boundary value problem. Fract. Calc. Appl. Anal. 16 978-984 (2013)

14. Ferreira, R.A.C.: On a Lyapunov-type inequality and the zeros of a certain Mittag-Leffler function. J. Math. Anal. Appl. 412, 1058-1063 (2014)

15. Ferreira, R.A.C.: Positive solutions for a class of boundary value problems with fractional $q$-differences. Comput. Appl. Math. 61, 367-373 (2011)

16. El-Shahed, M., Al-Askar, F.: Positive solutions for boundary value problem of nonlinear fractional q-difference equation. J. Math. Anal. Appl. 2011, 1-12 (2011)

17. Liang, S., Zhang, J.: Existence and uniqueness of positive solutions for three-point boundary value problem with fractional q-difference. J. Comput. Appl. Math. 40, 277-288 (2012

18. Jleli, M., Samet, B.: A Lyapunov-type inequality for a fractional q-difference boundary value problem. J. Nonlinear Sci. Appl. 9, 1965-1976 (2016)

19. Ma, K.K., Han, Z.L.: Lyapunov-type inequalities on fractional q-difference Schrodinger equation with the Woods-Saxon potential. Int. J. Dyn. Syst. Differ. Equ. (2018) (to appear)

20. Agarwal, R.P.: Certain fractional q-integrals and q-derivatives. Math. Proc. Camb. Philos. Soc. 66, 365-370 (1969)

21. Marinkovic, R.P.: Fractional integrals and derivatives in q-calculus. Appl. Anal. Discrete Math. 1, 311-323 (2007)

22. Annaby, M.H., Mansour, Z.S.: q-Fractional Calculus and Equations. Springer, Berlin (2012) 